NASA Tech Briefs announce new technology derived from the U.S. space program. They are issued to encourage commercial application. Tech Briefs are available on a subscription basis from the Clearinghouse for Federal Scientific and Technical Information, Springfield, Virginia 22151. Requests for individual copies or questions relating to the Tech Brief program may be directed to the Technology Utilization Division, NASA, Code UT, Washington, D.C. 20546.

\title{
Thermodielectric Radiometer
}

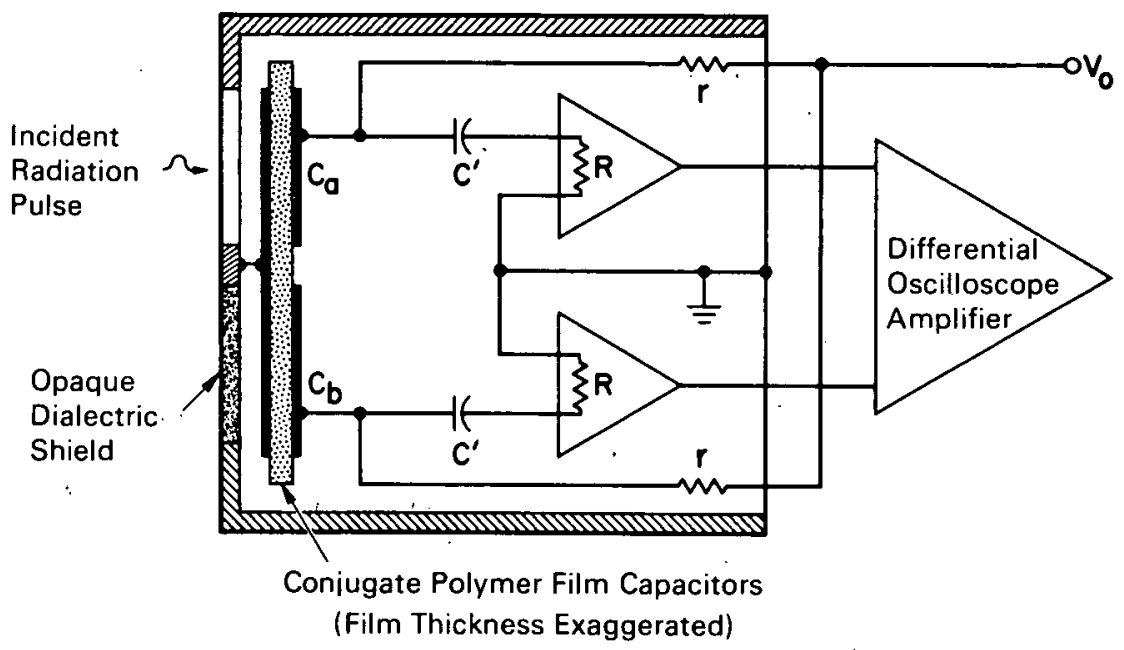

Conjugate Capacitor Configuration with Integral Preamplifiers

\section{The problem:}

To develop a radiometer with a wide and rapid spectral response for measuring microsecond pulses of radiant flux in the presence of the electromagnetic noise characteristically generated by spark gaps or shock tubes.

The solution:

A detector consisting of a charged capacitor that delivers a voltage pulse proportional to the thermally induced depolarization of its polymeric dielectric. How it's done:

A conjugate capacitor configuration with integral preamplifiers is shown in the figure. The common grounded electrode of the polymer-film dual capacitor $\left(C_{a}, C_{b}\right)$ is formed by evaporating a thin black gold deposit on an aluminum base that has been evaporated on a quarter-mill (6-micrometer) polymer film (e.g., Mylar). The microporous gold deposit has an absorption of at least 0.90 from the ultraviolet region to about 15 micrometers, giving the detector a wide: band sensitivity to radiant energy. The aluminum film is grounded by contact with the metal case, which acts as an electrostatic shield for the assembly. The individual electrodes on the other side of the polymer film are also of deposited aluminum metal and form 'two equal-valued capacitors of about $100 \mathrm{pF}$, sharing the same dielectric and ground electrode. Contact with the capacitors is made by a copper pellet at convenient points. The dual capacitor assembly is charged through current-limiting resistors (from a voltage source $\dot{V}_{0}$ ) to a gradient not exceeding the dielectric strength of the film, but generally of the order of about 100 volts/micrometer. Capacitors $C^{\prime}$ are used to couple signals to high-input impedance 
preamplifiers externally connected to a differentialinput oscilloscope amplifier.

Capacitor $\mathrm{C}_{\mathrm{a}}$ receives the incident radiation pulse and prevalent noise (e.g., electromagnetic noise accompanying a shock wave). Capacitor $C_{b}$ receives only the noise because the radiation pulse is blocked by an opaque dielectric shield. The signal from each capacitor is amplified by its preamplifier and transferred to the input terminals of the differential oscilloscope amplifier. If the two capacitors, $\mathrm{C}_{\mathrm{a}}$ and $\mathrm{C}_{\mathrm{b}}$, and their response to common-mode noise are identical, and if the preamplifiers are balanced, only the radiant energy signal is transferred by the oscilloscope amplifier.

Calibration can be effected with a $50 \mathrm{~mW}$, cw laser chopped at $1 \mathrm{kHz} / \mathrm{sec}$; the laser is referenced to a commercial, low-frequency radiometer. Typically, the radiometers have a sensitivity of $20 \mathrm{~V} /$ joule $/ \mathrm{cm}^{2}$ for a 6-micrometer film charged from a $500-\mathrm{V}$ source. Response times are a function of the thermal response of the polymer film, the electrical response of the detector's equivalent circuit, and the external readout circuit; response times of the order of several microseconds are readily achieved.

A radiometer utilizing only one radiation-sensitive capacitor can be constructed for use in a noise-free ambient environment. It can be completely potted with a dielectric compound if it is to be used at low ambient pressures where shorting caused by ionization can occur.

Notes:

1. Additional information is contained in the paper: Russell, L. D. and Beam, B. H.: Thermodielectric Radiometer, 68-403 presented at AIAA Third Aerodynamic Testing Conference, San . Francisco, Calif., April 8-10, 1968.

2. Requests for further information may be directed to:

$$
\begin{aligned}
& \text { Technology Utilization Officer } \\
& \text { Ames Research Center ' } \\
& \text { Moffett Field, California } 95014 \\
& \text { Reference: B70-10056 }
\end{aligned}
$$

\section{Patent status:}

Inquiries concerning rights for the commercial use of this invention may be made to National Aeronautics and Space Administration, Code GP, Washington, D.C. 20546

Source: Benjamin H. Beam and Larry D. Russell Ames Research Center (ARC-10138) 